\title{
Experimental Investigation of Laser Emission of Dye-Doped Cholesteric Liquid Crystals with a Cholesteric Reflector
}

\author{
Ying Zhou \\ Yuhua Huang \\ Alexandra Rapaport \\ Michael Bass \\ Shin-Tson Wu \\ College of Optics and Photonics, University of Central Florida, \\ Orlando, FL
}

\begin{abstract}
Dye-doped cholesteric liquid crystal (CLC) behaves like a one-dimensional photonic crystal laser when pumped by a second harmonic Nd-YAG pulsed laser. Usually circularly polarized laser light in the same sense as the cholesteric helix is emitted from both directions of the lasing cell. In this paper, we experimentally demonstrate the laser emission enhancement and investigate the corresponding polarization state from the dye-doped CLC laser in stack with another CLC reflector. Cell gap and dye concentration are important factors affecting the optical efficiency of the CLC lasers.
\end{abstract}

Keywords: cholesteric liquid crystal laser; cholesteric liquid crystal reflector; laser emission; laser enhancement

\section{INTRODUCTION}

Cholesteric liquid crystal (CLC) is a promising chiral photonic medium with one-dimensional periodic refractive index modulation. It has attracted numerous interests because of its potential applications in low threshold lasers, optical diodes, and optical amplifiers [1]. With the presence of photonic band gap, CLC is particularly useful in shaping the density of states, localizing photons, and guiding the waves. Due to Bragg reflection, the self-organized chiral structure shows a selective reflection for circularly polarized light when the light passes

Address correspondence to Shin-Tson Wu, College of Optics and Photonics, University of Central Florida, Orlando, FL 32816. E-mail: swu@mail.ucf.edu 
through the medium. Near the photonic band edge (PBE), group velocity is reduced substantially. Correspondingly, both density of states (DOS) and spontaneous emission rate which are inversely proportional to group velocity increase. Therefore, within the reflection band photon suppression occurs while at PBE photon enhancement occurs. This enhancement has been extensively exploited through chiral LC host incorporated with fluorescent dyes to generate stimulated emission. Chiral photonic structure of CLC works as a distributed feedback cavity for band-edge resonant mode.

Following the first experimental demonstration [2] and theoretical prediction [3] of photonic band edge lasing from dye-doped CLC, tremendous efforts have been concentrated on the development of low threshold and tunable lasing action with high efficiency. So far, reports have shown that low threshold laser action are readily observed not only in chiral nematic liquid crystal [4,5] but also in ferroelectric liquid crystal (smectic $\mathrm{C}^{*}$ ) [6], blue phase liquid crystal [7], cholesteric polymer [8,9], cholesteric elastomer [10] and more recently cholesteric glasses [11]. Tunability of lasing in these systems can be achieved by the control of chiral agent concentration, external electrical field [12], temperature [13], mechanical stress [10], and photochemical effect $[14,15]$.

In addition, there also have been a lot of reports dedicated to the study of lasing performance, or optical efficiency for dye-doped CLC laser in order to find the best lasing condition from a material viewpoint. Results suggest that laser dye's order parameter of the transition dipole moment for emission $\left(\mathrm{S}_{\mathrm{T}}\right)$ and orientational order parameter play key roles affecting the emission. Lasing is more favorable at the band edge where $\mathrm{S}_{\mathrm{T}}$ is along local director [16]. Polymeric dyes have been applied to enhance the order parameter and an optical efficiency as high as $25 \%$ has been achieved [17].

For a CLC-based distributed feedback laser, usually laser emission comes out from both sides of the lasing cell due to the symmetry of cavity. In this paper, we demonstrate laser emission enhancement from one side of the lasing cell which is in proximity contact with another CLC reflector. We focus our attention on the optimization of laser cavity from a device viewpoint rather than a material viewpoint. Based on the current material, different thicknesses of lasing cell and different dye concentrations are investigated with a CLC reflector attached in order to achieve the optimal photon confinement and, in the mean time, avoid the photo quenching effect of dyes.

A CLC mixture composed of nematic liquid crystal and chiral agent shows a selective reflection band for the circularly polarized light as a consequence of helical molecular assemblages [18]. When the linearly 
polarized light propagates into the CLC medium, within the reflection band the circularly polarized light in the same sense with CLC will be totally reflected and the light in the opposite sense will be totally transmitted, assuming that the number of periodicity is enough for Bragg reflection. The center wavelength $\lambda_{0}$ and bandwidth $\Delta \lambda$ of the reflection band are determined by the following equations:

$$
\begin{aligned}
\lambda_{0} & =\langle n\rangle p, \\
\Delta \lambda & =\Delta n p,
\end{aligned}
$$

where $\langle n\rangle, \Delta n$ and $p$ denote the average refractive index, birefringence of the nematic LC, and the intrinsic pitch length of the CLC. The pitch length is related to helical twist power of the chiral dopant (HTP) and the weight concentration $(\mathrm{c} \%)$ through

$$
p=\frac{1}{H T P \cdot c \%} .
$$

\section{EXPERIMENTS AND RESULTS}

In experiment, we prepared a right-handed CLC host by mixing $27.3 \mathrm{wt} \%$ Merck chiral dopant MLC6248 $\left(\mathrm{HTP}=11 \mu \mathrm{m}^{-1}\right)$ in a positive dielectric anisotropic Merck nematic LC host BL006 ( $\Delta n=0.286)$. A $1.5 \mathrm{wt} \%$ laser dye (4-(dicyanomethylene)-2-methyl-6(4-dimethlyaminostryl)-4H-pran) (DCM, from Exciton) was dissolved into the CLC host. Lasing samples in $8 \mu \mathrm{m}, 10 \mu \mathrm{m}$ and $15 \mu \mathrm{m}$ cell gaps were fabricated. The inner surface of the glass substrate was coated with a thin indium-tin-oxide (ITO) layer $(\sim 100 \mathrm{~nm})$ and then overcoated with a thin polyimide layer $(\sim 80 \mathrm{~nm})$. The polyimide layer was rubbed in anti-parallel direction to typically generate $a \sim 3^{\circ}$ pretilt angle. Another CLC cell without dye was used as a right-handed circularly polarized reflector. To make a CLC reflector, we doped $25 \mathrm{wt} \%$ MLC6248 into BL006 in a $5 \mu \mathrm{m}$ homogeneous cell. Its band gap was chosen to cover the lasing wavelength. During fabrication, the whole mixture was stirred thoroughly and then capillary-filled into LC cells in an isotropic state. A slowly cooling process is necessary to obtain a relatively uniform cholesteric planar (Grandjean) structure. However due to the weaker anchoring energy in a thick cell (e.g., $15 \mu \mathrm{m}$ ), multi-domain textures leading to light scattering cannot be completely eliminated.

Figure 1 shows the experimental setup at the normal incidence. A frequency-doubled Nd-YAG pulsed laser $(\lambda=532 \mathrm{~nm}, 4 \mathrm{~ns}$ pulse width, and $1 \mathrm{~Hz}$ repetition rate) was used as pumping source. The incident 


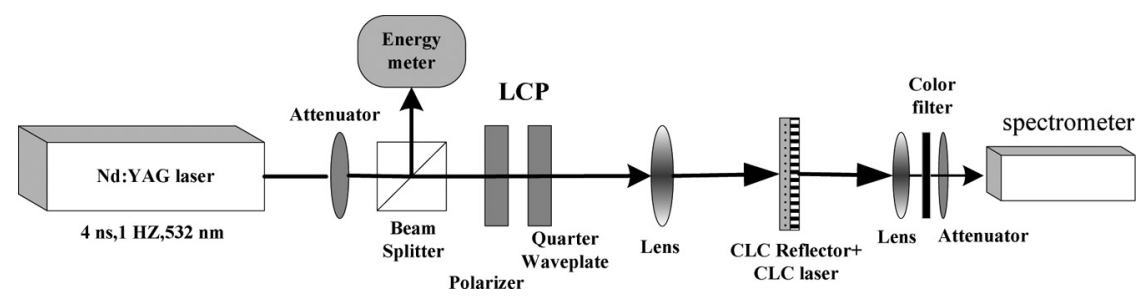

FIGURE 1 Experimental set up with normal incidence.

laser beam was separated by a beam splitter into two paths: one was monitored by an energy meter (Ophir, Laserstar) and the other was converted to a left-handed circularly polarized beam before being focused $(\sim 200 \mu \mathrm{m}$ in diameter) onto the lasing cell. The left-handed polarized pumping beam can be transmitted into the lasing sample which has right-handed helix even if the reflection band covers the pump wavelength. The reflector was attached to the lasing sample in proximity contact on the side facing toward the pump beam. No index matching fluid and no anti-reflection coating were used between the cell substrates. The Fresnel reflection from index mismatch of the substrates and the air-substrate surfaces brings in: 1) a small part of opposite circularly polarized light component from what can be generated from CLC laser, and 2) The reflector and the lasing cell have to be well attached in order to observe the enhancement phenomena otherwise it becomes the loss in the cavity and no dramatic enhancement can be observed. With a right handed helix, the CLC reflector only reflects the CLC laser light (right-handed circular polarization, RCP) while transmits the pump beam (left-handed circular polarization, LCP). Laser emission from the dye-doped CLC cell was then focused to a fiber-based spectrometer (HR2000, Ocean Optics) along the direction perpendicular to the glass substrates.

Figure 2 shows the reflection band of the dye-doped CLC lasing cell at 8,10 , and $15 \mu \mathrm{m}$ cell gaps. As an example, the original laser emission spectrum from the $8 \mu \mathrm{m}$ CLC laser pumped at $30 \mu \mathrm{J} /$ pulse is included in Figure 2 as well. The laser action centered at $\lambda \sim 602 \mathrm{~nm}$ takes place at the low energy band edge of the dye-doped CLC laser. The measured full width of half maximum (FWHM) is $\sim 0.8 \mathrm{~nm}$. The high energy band edges of the three CLC lasing cells are completely obscured by the DCM absorption. Reflection band of the CLC reflector is chosen to reflect the lasing wavelength.

Figures 3 to 5 plot the original laser emission from the dye-doped CLC laser, laser emission enhanced by the CLC reflector, and their 


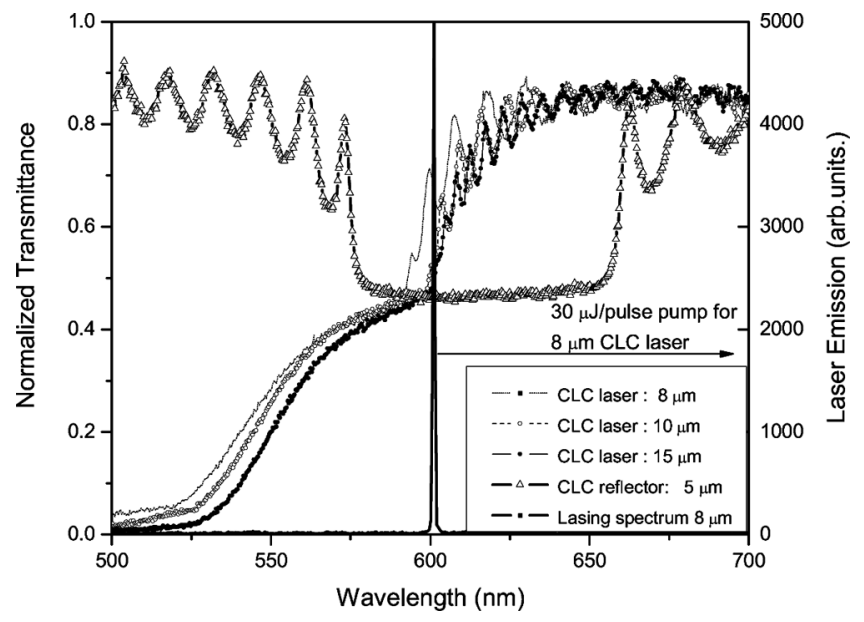

FIGURE 2 Reflection band of dye-doped CLC lasers at $8 \mu \mathrm{m}, 10 \mu \mathrm{m}, 15 \mu \mathrm{m}$, reflection band of the CLC reflector and the lasing spectrum for $8 \mu \mathrm{m}$ CLC laser pumped by $\mathrm{Nd}$ :YAG frequency-doubled pulsed laser at $30 \mu \mathrm{J} /$ pulse, $532 \mathrm{~nm}, 1 \mathrm{~Hz}$ and $4 \mathrm{~ns}$.

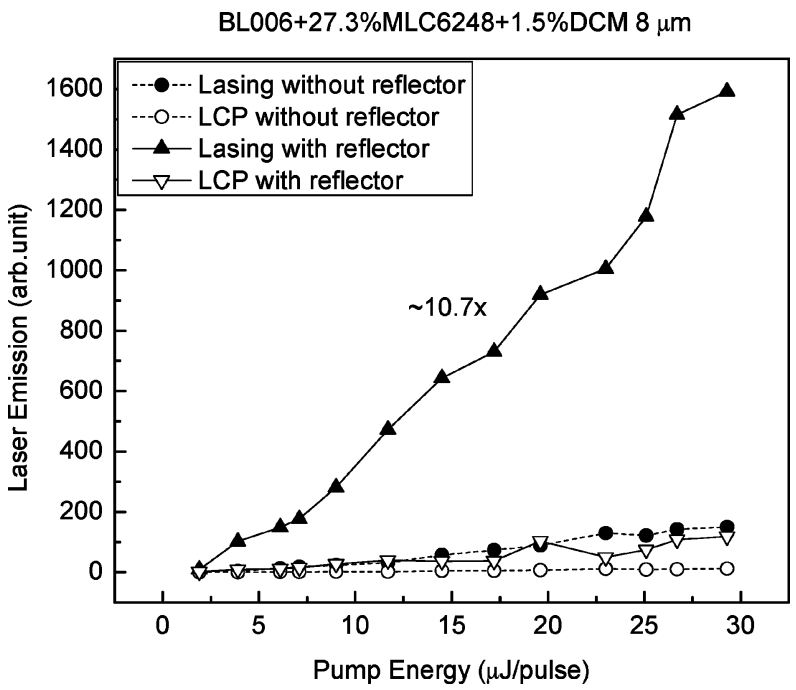

FIGURE 3 Pump energy dependence of laser emission from an $8 \mu \mathrm{m}$ dye doped CLC laser with a CLC reflector and the corresponding LCP component. 


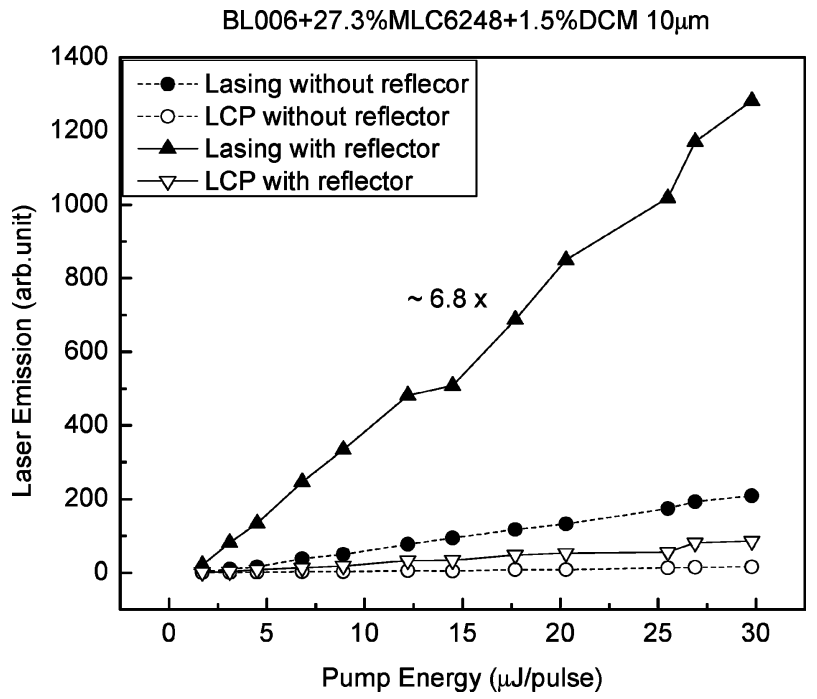

FIGURE 4 Pump energy dependence of laser emission from a $10 \mu \mathrm{m}$ dye doped CLC laser with a CLC reflector and the corresponding LCP component.

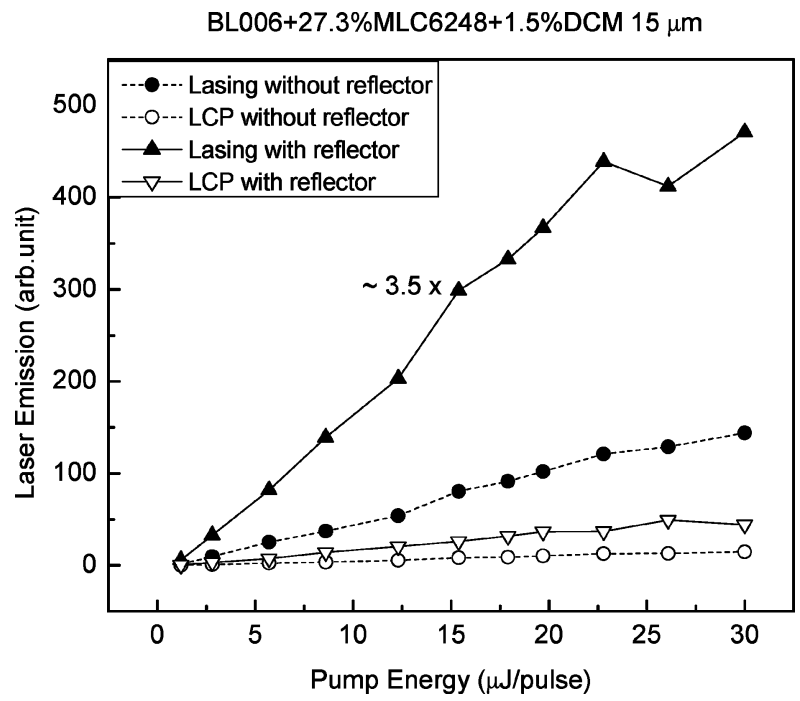

FIGURE 5 Pump energy dependence of laser emission from a $15 \mu \mathrm{m}$ dye doped CLC laser with a CLC reflector and the corresponding LCP component. 
corresponding LCP components for the 8,10 , and $15 \mu \mathrm{m}$ lasing cells. The lasing threshold was measured to be $1.8,1.0$, and $0.5 \mu \mathrm{J} /$ pulse and the lasing wavelength was 602,603 , and $604 \mathrm{~nm}$ for the 8,10 , and $15 \mu \mathrm{m}$ cells, respectively. In Figures 3-5, lines with filled triangles and filled circles represent the total laser emission with and without a CLC reflector. Lines with open triangles and open circles represent the LCP components with and without a CLC reflector. The numbers inside the figure show the average enhancement ratio at different pumping energy. The fluctuation for the enhanced laser emission originates from the pump laser's intensity fluctuation and physically different reflection point on the CLC reflector when each pulse was captured.

For the original laser emission when no CLC reflector is attached, the laser light is mainly circularly polarized in the same sense as the CLC helix of the lasing cell. For our right handed CLC lasers, RCP occupies $\sim 90 \%$ and LCP occupies $\sim 10 \%$ of the total output. The small amount of opposite circularly polarized component (LCP) is believed to originate from the imperfect cholesteric structures. Covering the lasing wavelength, the CLC reflector in stack with the lasing cell dramatically enhances the original laser emission, which is readily observed in all lasing cells with different cell gaps. Unlike the traditional metallic mirror, a CLC reflector reflects the laser beam without $\pi$ phase change and hence the polarization state of the reflected laser beam (RCP) is preserved. As a polarization-dependent distributed feedback cavity facilitating the RCP band edge mode only, the lasing cell further amplifies the reflected beam which was not supposed to transmit through the laser cavity. This can be supported by the fact that the enhancement ratio in all three lasing samples is more than doubled, considering the single-sided emission versus the doublesided emission. The CLC reflector, in this scenario, functions equivalently as a "mirror" for this polarization selective feedback cavity and the effective length of amplifier is consequently doubled.

It is also noted that the enhancement ratio decreases as the thickness of lasing cell increases. This is believed to be attributed to the difference of net gain increase for different thicknesses of dye-doped CLC layer. Below the saturation length (determined by the population inversion between uper and lower energy levels) the laser intensity grows exponentially with the gain length. As doubling the effective amplifier length by means of a CLC reflector, gain and loss are simultaneously doubled. The enhanced ratio is exponentially proportional to the net gain increase, or the difference between the gain and loss. Scattering loss inside the cavity caused by non-uniform cholesteric multi-domains in thinner cells are much smaller than that in the thicker cell, due to the stronger anchoring force in thinner cells. 
On the other hand, the galss substrate between the lasing cell and the reflector also plays important role in the further amplification process. The thinner cell provides larger acceptance angle for the reflected light due to a larger divergence. Hence in a thinner cell the reflected light is easier to get back into the original pump area to be further amplified. These two factors balance each other and the best lasing performance with CLC reflector enchanced was found in the 8 or $10 \mu \mathrm{m}$ cell (their performance is close to each other) where single domain is barely retained.

Concentration effect of DCM was studied as well. Figure 6 shows the pump energy dependence of lasing performance enhanced by a CLC reflector at dye concentrations from $0.5 \mathrm{wt} \%$ to $2 \mathrm{wt} \%$. In Figure 6 , lines in filled circles, filled triangles, filled squares, and filled stars represent the laser emission enhanced by a CLC reflector. Lines in open circles, open triangles, open squares, and open stars represent the original laser emission without a CLC reflector. Without the CLC reflector, the best lasing performance obtained is at $1.5 \%$ DCM, followed by $1 \%, 2 \%$ and $0.5 \%$. With the reflector, the highest efficiency is still found at $1.5 \%$ DCM, followed by $1 \%, 2 \%$ and $0.5 \%$. The results of $1 \%$ DCM and $2 \%$ DCM are almost the same, which means for $2 \%$ concentration, the quenching effect already takes place so that the lasing efficiency tends to saturate.

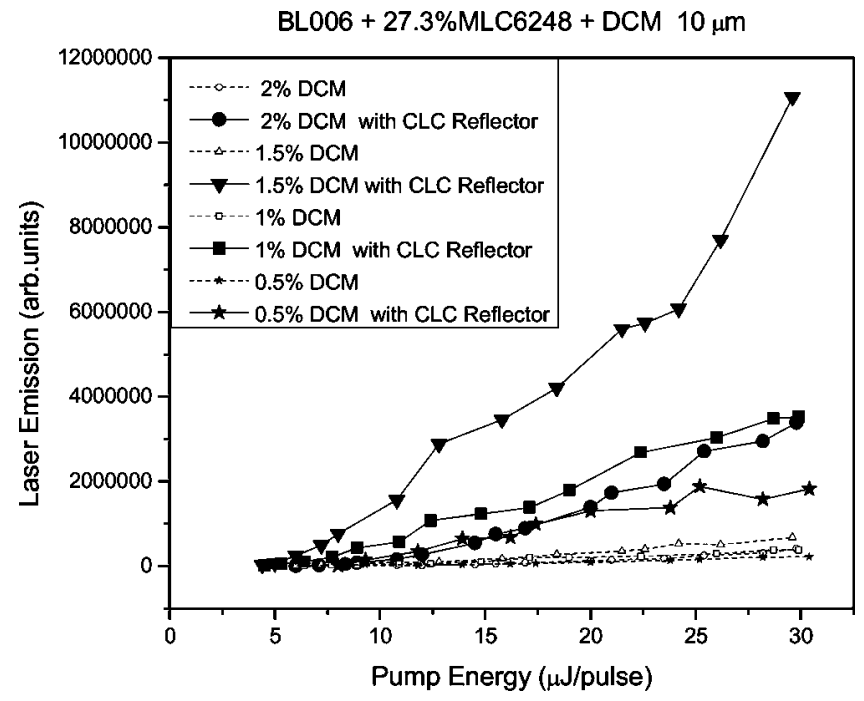

FIGURE 6 Pump energy dependence of laser emission enhanced by a CLC reflector at dye concentration of $0.5 \%, 1 \%, 1.5 \%$ and $2 \%$. 


\section{CONCLUSION}

We have developed a simple CLC reflector method for doubling the optical efficiency of a dye-doped CLC laser. Emission enhancement effect for different cell gaps and different dye concentrations were investigated where the best lasing performance enhanced by CLC reflector was found in the $10 \mu \mathrm{m}$ CLC lasing cell and $1.5 \mathrm{wt} \%$ laser dye (DCM). Theoretical simulation for understanding the observed phenomenon is under way.

\section{REFERENCES}

[1] Kopp, V. I., Zhang, Z. Q., \& Genack, A. Z. (2003). Progress in Quantum Electronics, $27,369$.

[2] II'Chishin, I. P., Tikhonov, E. A., Tishchenko, V. G., \& Shpak, M. T. (1980). JETP Lett., 32, 24.

[3] Dowling, J. P., Scalora, M., Bloemer, M. J., \& Bowden, C. M. (1994). J. Appl. Phys., $75,1896$.

[4] Kopp, V. I., Fan, B., Vithana, H. K. M., \& Genack, A. Z. (1998). Opt. Lett., 23, 1707.

[5] Lin, S. Y., Fleming, J. G., \& Eikady, I. (2003). Appl. Phys. Lett., 83, 593.

[6] Ozaki, M., Kasano, M., Ganzke, D., Haase, W., \& Yoshina, K. (2002). Adv. Mater., $14,306$.

[7] Cao, W. Y., Munoz, A., Palffy-Muhoray, P., \& Taheri, B. (2002). Nat. Mater., 1, 111.

[8] Matsui, T., Ozaki, R., Funamoto, K., Ozaki, M., \& Yoshino, K. (2002). Appl. Phys. Lett., 81, 3741.

[9] Ohta, T., Song, M. H., Tsunoda, Y., Nagata, T., Shin, K. C., Araoka, F., Takanishi, Y., Ishkawa, K., Watanabe, J., Nishimura, S., Toyooka, T., \& Takezoe, H. (2004). Jpn. J. Appl. Phys., 43, 6142.

[10] Finkelmann, H., Kim, S. T., Munoz, A., Palffy-Muhoray, P., \& Taheri, B. (2001). Adv. Mater., 13, 1069.

[11] Hibaev, P. V., Opp, V., Enack, A., \& Anelt, E. (2003). Liq. Cryst., 30, 1391.

[12] Furumi, S., Yokoyama, S., Otomo, A., \& Mashiko, S. (2003). Appl. Phys. Lett., 82, 16.

[13] Moreira, M. F., Carvalho, I. C. S., Cao, W., Bailey, C., Taheri, B., \& Palffy-Muhoray, P. (2004). Appl. Phys. Lett., 85, 2691.

[14] Chanishvili, A., Chilaya, G., Petriashvili, G., Barberi, R., Bartolino, R., Cipparrone, G., Mazzulla, A., \& Oriol, L. (2003). Appl. Phys. Lett., 83, 5353.

[15] Fuh, A. Y. G., Lin, T. H., Liu, J. H., \& Wu, F. C. (2004). Opt. Express, $12,1857$.

[16] Morris, S. M., Ford, A. D., Pivnenko, M. N., \& Coles, H. J. (2005). J. Appl. Phys., 97, 023103.

[17] Araoka, F., Shin, K. C., Takanishi, Y., Ishikawa, K., Takezoe, H., Zhu, Z. G., \& Swager, T. M. (2003). J. Appl. Phys., 94, 279.

[18] Wu, S. T. \& Yang, D. K. (2001). Reflective Liquid Crystal Displays, Wiley: New York. 